\title{
Modelling of magnetic field and flux in objects having surface-hardening and transition layer
}

\author{
Vladimir Kostin ${ }^{1,2, *}$, Olga Vasilenko $^{1,2}$, and Alexander Byzov ${ }^{1}$ \\ ${ }^{1}$ IMP UB RAS, Laboratory of Integrated Control Methods, 620108 Ekaterinburg, Russia \\ ${ }^{2} \mathrm{UrFU}$, Institute of Physics and technology, 620002, Ekaterinburg, Russia
}

\begin{abstract}
A finite element simulation of the distribution of the magnetic field and flux in locally magnetized steel objects subjected to surface hardening and having after hardening a three-layer structure: a hardened surface layer, a transition layer, an unstressed core; has been conducted. Magnetization of the tested object was conducted using a U-shaped electromagnet. As a result, pictures of the distribution of the magnetic field in the monitoring object were obtained. The values of induction depending on the depth of the hardened layer for a fixed transition layer at different points of space relative to the surface of the object of control are obtained.
\end{abstract}

\section{Introduction}

Surface hardening of steel products is conducted to increase their wear resistance and fatigue failure resistance. The main types of surface hardening include surface hardening of steel using various methods of heating the product (flame, electrolytes, molten metals and salts, laser, high and industrial frequency currents); chemical-thermal treatment of steel (cementation, nitration, cyanidation), hardening by the method of surface-plastic deformation [1].

To control the quality of surface hardening, magnetic control methods are widely used. To understand the processes occurring when magnetizing massive layered objects and for expanding the range of controlled products, including for creating methods for controlling massive objects by the properties of matter, it is necessary to investigate the spatial distribution of the field and the magnetic flux both in the controlled object and over its surface. Systems with attached magnetizing devices are widely used, and the most common devices are U-shaped electromagnets in magnetic nondestructive testing.

There are experimental and theoretical methods for studying and optimizing composite magnetic circuits. In order to study the spatial distribution of the magnetic field and the flow inside and near the objects of control, experimental methods can be used very narrowly. Theoretical investigation of magnetic circuits and transducers implies a solution of the system of Maxwell's equations. To solve this problem, the most accessible method is $3 \mathrm{~d}$ modeling, which consists in the numerical solution of the system of Maxwell equations for given boundary conditions. Computer modeling is now an important part of the process

* Corresponding author: kostin@imp.uran.ru 
of designing devices of various types, which is due to the need to shorten the development of new devices, as well as the existence of effective algorithms and programs for computer simulation of electrical and magnetic circuits $[2,3]$.

\section{Experimental}

\subsection{Description of the model experiment}

All the calculations which were carried out in the ANSYS CFX Academic Research finite element modeling software in our study was performed using «Uran» supercomputer of IMM UB RAS. The calculation model was an object consisting of three layers: a hardened material (a magnetically hard material with a magnetic permeability $\mu=95 \mathrm{G} / \mathrm{m}$ and a coercive force $\mathrm{H}_{\mathrm{c}}=2400 \mathrm{~A} / \mathrm{m}$ ), a transition layer (material - steel 35) and a core (soft magnetic material - steel 3); and a U-shaped electromagnet (Armco-iron material), the tested object with dimensions $56 \times 300 \times 230 \mathrm{~mm}$.

The thicknesses of the hardened and transition layers were variable quantities. The model of the electromagnet had an inter-pole distance of $30 \mathrm{~mm}$, and the cross-section of the poles was $12 \times 28 \mathrm{~mm}$. The computational model of a massive three-layered object, locally magnetized by a U-shaped electromagnet, is shown in Fig. 1

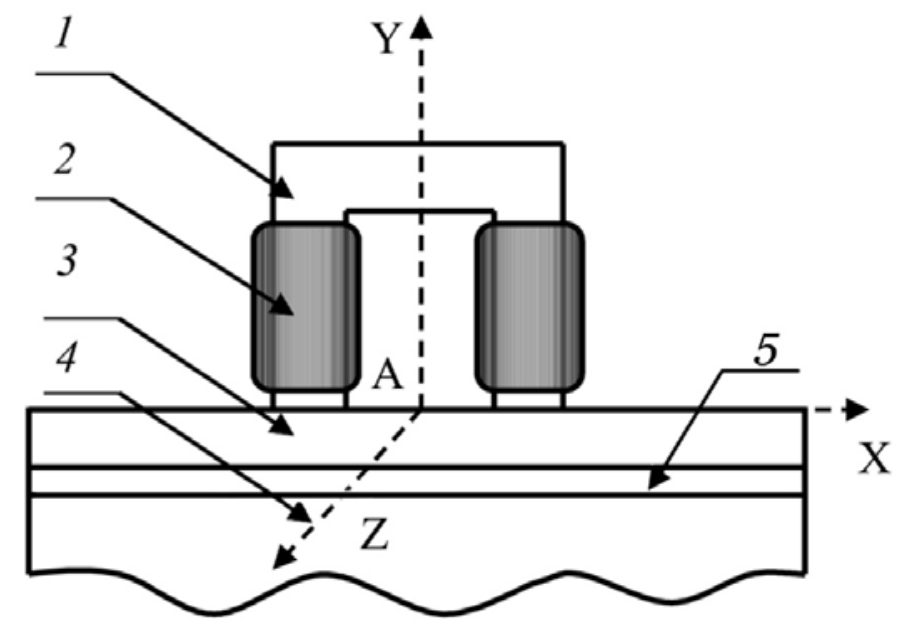

Fig.1. Calculation model of a massive three-layered object locally magnetized by a U-shaped electromagnet (1 - magnet yoke, 2 - magnetization winding, 3 - hardened layer, 4 - core, 5 - transition layer)

When setting up model experiments, the value of the hardened layer varied within 0.5$20 \mathrm{~mm}$; the thickness of the transition layer was $2 \mathrm{~mm}$; the magnitude of the magnetomotive force in the magnetic circuit was $1800 \mathrm{~A}$.

\subsection{Modeling}

As a result of the model calculations, the following data were obtained.

Figure 2 shows the distribution of the magnetic induction in the test object near the electromagnet pole with a thickness of the hardened layer of $20 \mathrm{~mm}$, a transition layer of 2 $\mathrm{mm}$, a core of $208 \mathrm{~mm}$ and a magnetomotive force of $1800 \mathrm{~A}$. 
In carrying out such experiments, it is important to show how the strength of the magnetic field varies depending on the parameters of the object and the conditions for monitoring. Calculations were made of the distribution of the intensity at a magnetomotive force of $1800 \mathrm{~A}$.

Figures 3 and 4 show the dependence of the magnetic strength on the depth of the hardened layer, respectively, at a height of $2 \mathrm{~mm}(\mathrm{~h}=2 \mathrm{~mm})$ from the control surface, at the surface of the test object $(\mathrm{h}=0 \mathrm{~mm})$, at a depth of $2 \mathrm{~mm}$ from the surface of the test object $(\mathrm{h}=-2 \mathrm{~mm})$ in the neutral plane of the computational model and in the middle between the neutral plane and the electromagnet pole (at a distance of $7.5 \mathrm{~mm}$ from the neutral plane).

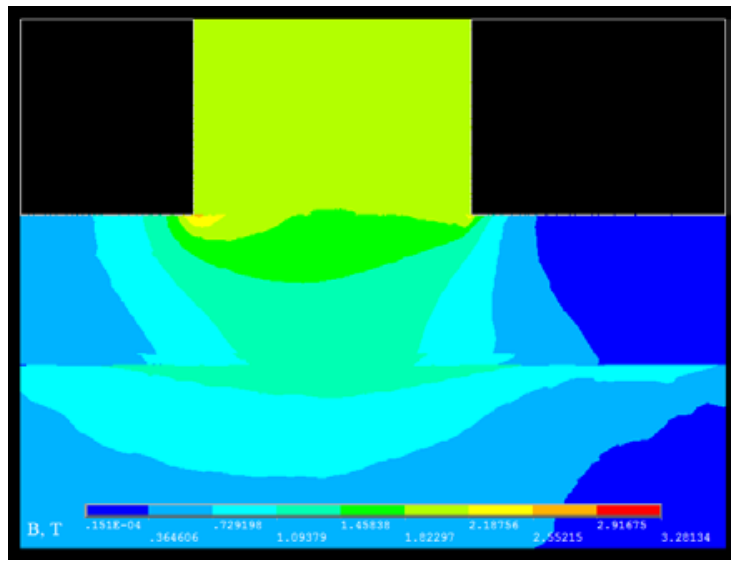

Fig. 2. Distribution of magnetic induction in a test object near the pole of an electromagnet

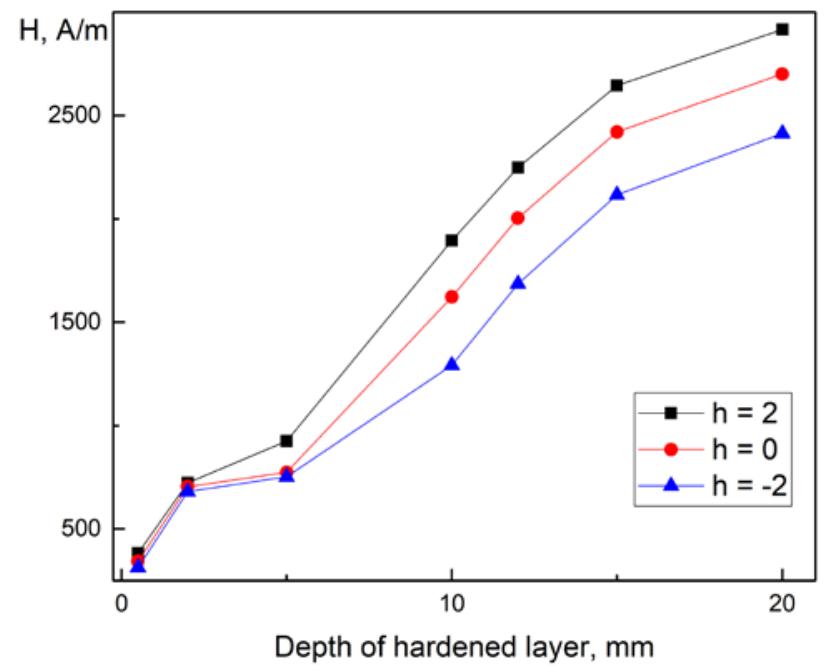

Fig. 3. Dependence of the magnetic field strength on the thickness of hardened layer at points $h=$ $2 \mathrm{~mm}, \mathrm{~h}=0 \mathrm{~mm}, \mathrm{~h}=-2 \mathrm{~mm}$ of the neutral plane of the electromagnet 


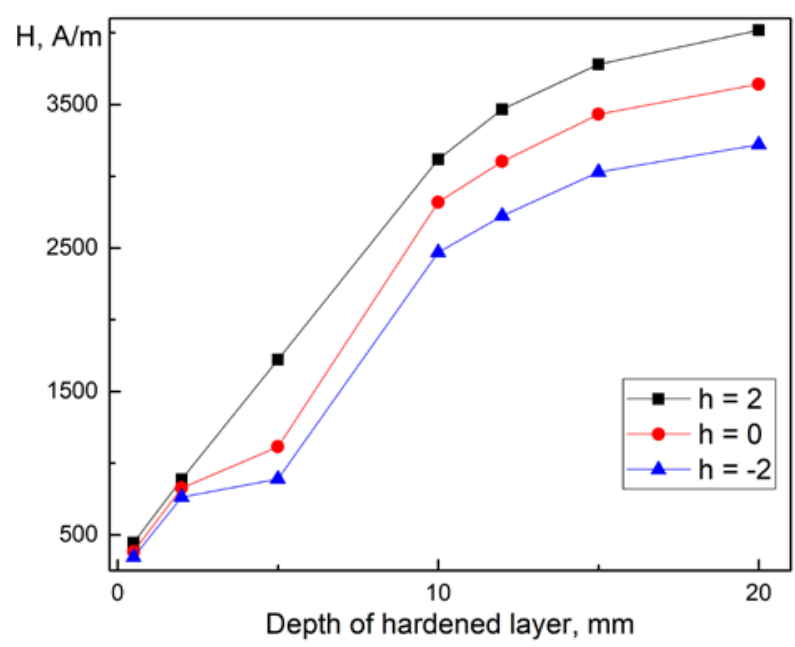

Fig. 4. Dependences of the magnetic field strength strength on the thickness of hardened layer at the points $\mathrm{h}=2 \mathrm{~mm}, \mathrm{~h}=0 \mathrm{~mm}, \mathrm{~h}=-2 \mathrm{~mm}$ at a distance of $7.5 \mathrm{~mm}$ from the neutral plane of the electromagnet

\section{Discussion}

It can be seen from Fig. 3, that the magnetic field strength above the surface and under the surface increases monotonically and is proportional, this indicates that by measuring the strength of the magnetic field on the surface of a layered object, it is possible to control the depth of the hardened layer by fixing the value of the magnetic flux. From the obtained dependence, it can be concluded that the range of controlled depths of the quenched layer can vary from 0.5 to $15 \mathrm{~mm}$ at a pole thickness of the magnetizing device of $12 \mathrm{~mm}$.

Figure 4 repeats the dependences of the magnetic field strength obtained from the depth of the quenched layer obtained for the neutral plane, however, the absolute values in this case are higher, and it indicates that the possible optimal location of the field sensor when determining the depth of the wound layer by the value of the intensity measured by the sensor.

\section{Conclusion}

As a result of the model study, the patterns of the distribution of magnetic fields and near the surface and inside the surface-hardened steel control object were obtained. It was found that the range of controlled thicknesses of the hardened layer is able to exceed the thickness of the poles, in contrast to the known coercimetric method.

According to the obtained dependences, it is possible to control the depth of the hardened layer when measuring the relative strength of the magnetic field on the surface of a layered object at a fixed value of the magnetic flux. It is shown that the optimal location of the field sensor is not the neutral plane, but the center between the neutral plane and the boundary of the inner surface of the electromagnet pole, which will be taken into account and investigated in further experimental verification of the results obtained.

The study has been conducted on the project "Diagnostics" №AAAA-A18-118020690196-3 


\section{References}

1. V.N.Kostin, Electromagnitniy control: uchebnoe izdanie (UrFU, IMP UB RAS, Ekaterinburg, 2016)

2. V.N. Kostin, O.N. Lukinykh, Y.G. Smorodinskii, K.V. Kostin, Simulation of field and inductance spatial distribution in locally magnetized massive objects and optimization of U-shaped transducer design, Russian Journal of Nondestructive Testing, v.6(46), pp. 403-410 (2010)

3. O.N. Vasilenko, V.N. Kostin, The topography of the field and flux inside and above the surfaces of ferromagnetic plates during their contact and contactless magnetization, Russian Journal of Nondestructive Testing, v.9(49), pp. 510-518 (2013) 